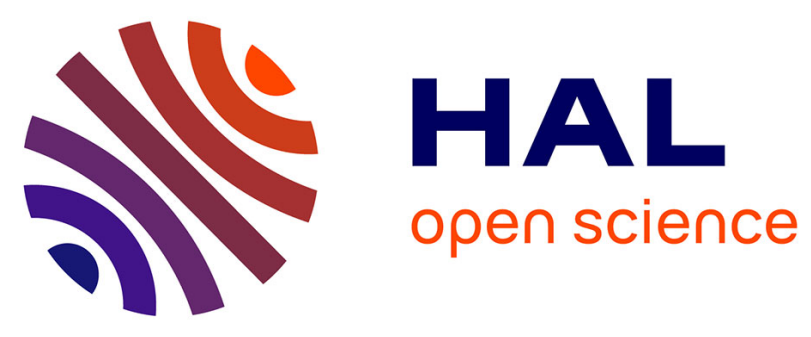

\title{
Prediction of Unruptured Intracranial Aneurysm Evolution: The UCAN Project
}

Vincent L'Allinec, Stéphanie Chatel, Matilde Karakachoff, Emmanuelle

Bourcereau, Zeineb Es-Salah-Lamoureux, Alban Gaignard, Florent

Autrusseau, Solène Jouan, Anne-Clémence Vion, Gervaise Loirand, et al.

\section{To cite this version:}

Vincent L'Allinec, Stéphanie Chatel, Matilde Karakachoff, Emmanuelle Bourcereau, Zeineb Es-SalahLamoureux, et al.. Prediction of Unruptured Intracranial Aneurysm Evolution: The UCAN Project. Neurosurgery, 2020. hal-02889889

\section{HAL Id: hal-02889889 https://hal.science/hal-02889889}

Submitted on 14 Oct 2021

HAL is a multi-disciplinary open access archive for the deposit and dissemination of scientific research documents, whether they are published or not. The documents may come from teaching and research institutions in France or abroad, or from public or private research centers.
L'archive ouverte pluridisciplinaire HAL, est destinée au dépôt et à la diffusion de documents scientifiques de niveau recherche, publiés ou non, émanant des établissements d'enseignement et de recherche français ou étrangers, des laboratoires publics ou privés. 


\section{Neurosurgery \\ Unruptured Cerebral Aneurysm, prediction of evolution: The UCAN Project \\ --Manuscript Draft--}

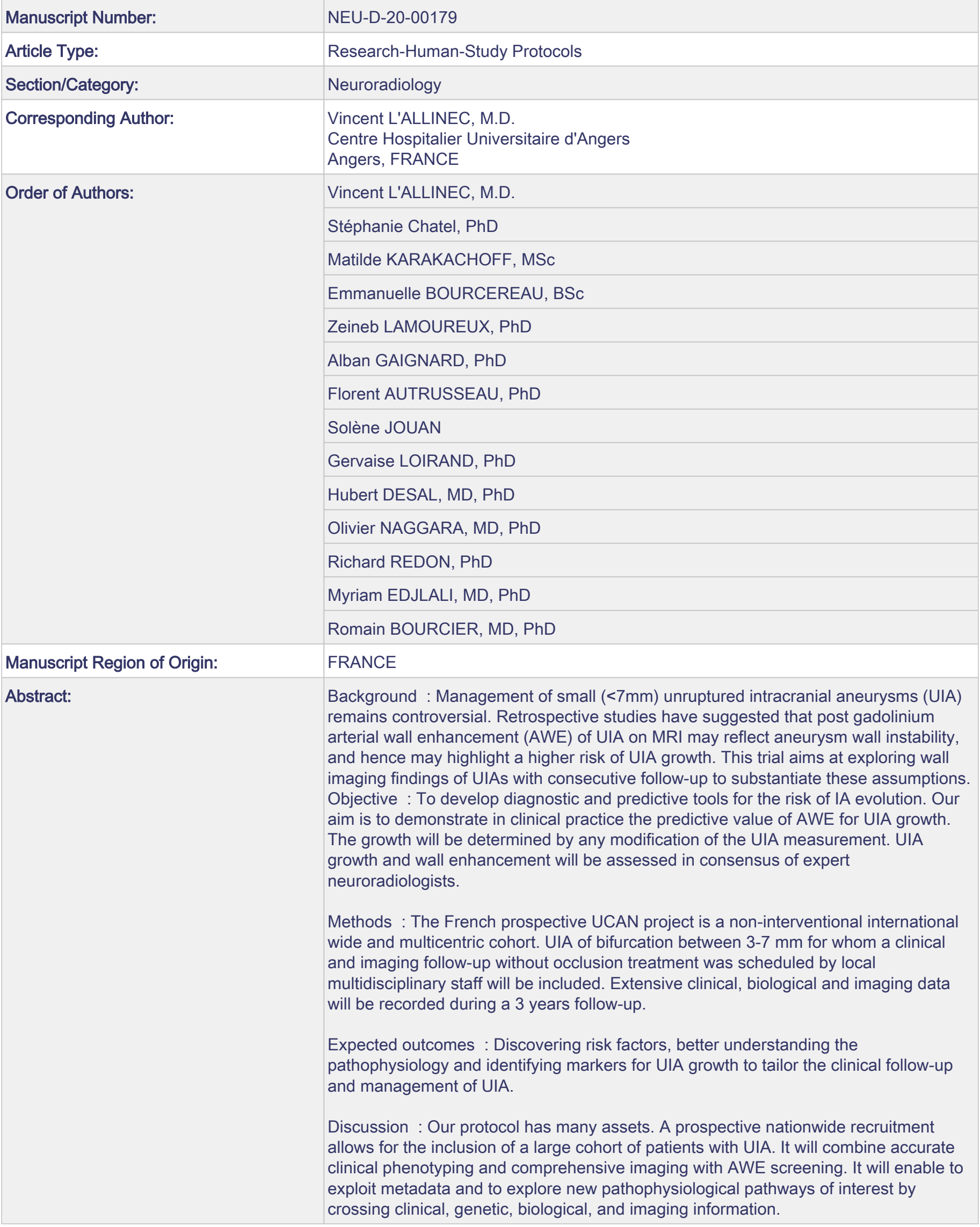




\begin{tabular}{|c|c|}
\hline \multirow[t]{2}{*}{ Suggested Reviewers: } & $\begin{array}{l}\text { PASQUALE MORDASINI } \\
\text { Insel } \\
\text { pasquale.mordasini@insel.ch } \\
\text { expert in the field }\end{array}$ \\
\hline & $\begin{array}{l}\text { M.D.I VERGOUWEN } \\
\text { Universiteit Utrecht } \\
\text { m.d.i.vergouwen@umcutrecht.nl }\end{array}$ \\
\hline \multicolumn{2}{|l|}{ Opposed Reviewers: } \\
\hline \multicolumn{2}{|l|}{ Additional Information: } \\
\hline $\begin{array}{l}\text { Compliance with Research Reporting } \\
\text { Guidelines: } \\
\text { Neurosurgery endorses several reporting } \\
\text { guidelines and requires authors to submit } \\
\text { their research articles in accordance with } \\
\text { the appropriate guideline statement(s) } \\
\text { and checklist(s). Completed applicable } \\
\text { checklists and flow diagrams must be } \\
\text { included with submissions. }\end{array}$ & $\begin{array}{l}\text { Yes - Submission Adheres to Appropriate Reporting Guideline(s) and Applicable } \\
\text { Checklists/Materials Are Included }\end{array}$ \\
\hline $\begin{array}{l}\text { Research articles that must be submitted } \\
\text { according to the appropriate reporting } \\
\text { guideline(s) include, but are not limited to: } \\
\text { randomized trials, systematic reviews, } \\
\text { meta-analyses of interventions, meta- } \\
\text { analyses of observational studies, } \\
\text { diagnostic accuracy studies, and } \\
\text { observational epidemiological studies (eg, } \\
\text { case series, cohort, case-control, and } \\
\text { cross-sectional studies). Consult the } \\
\text { EQUATOR Network, which maintains a }\end{array}$ & \\
\hline
\end{tabular}


useful, up-to-date list of guidelines as they are published, with links to articles and checklists: http://www.equatornetwork.org.

Please confirm below that information is reported according to the relevant reporting guideline(s) and any required materials are included with the submission:

Please indicate which reporting

guideline(s) the study adheres to (eg, STROBE, PRISMA, CONSORT). as follow-up to "Compliance with Research Reporting Guidelines:

Neurosurgery endorses several reporting guidelines and requires authors to submit their research articles in accordance with the appropriate guideline statement(s) and checklist(s). Completed applicable checklists and flow diagrams must be included with submissions.

Research articles that must be submitted according to the appropriate reporting guideline(s) include, but are not limited to: randomized trials, systematic reviews, meta-analyses of interventions, metaanalyses of observational studies, diagnostic accuracy studies, and observational epidemiological studies (eg, case series, cohort, case-control, and cross-sectional studies). Consult the EQUATOR Network, which maintains a useful, up-to-date list of guidelines as they are published, with links to articles and checklists: http://www.equatornetwork.org.

Please confirm below that information is reported according to the relevant reporting guideline(s) and any required materials are included with the submission:"

\section{Statistical Analysis:}

Not Applicable - Article does not report statistics.

For manuscripts that report statistics, the Editor requires that the authors provide evidence of statistical consultation or expertise.

If your article includes statistics, has the information reported been evaluated by 
an expert?

IRB/Ethics Approval:

Yes

Please indicate if your study has received institutional review board/ethics approval.

If yes, these materials are readily available should the Editor request them.

Funding:

Funding Agencies : This study was supported by a grant from the French Ministry of Study Protocols must have received grant Health (U-CAN, 2019, PHRC-19-0394)

funding from a major extramural funding body at the time of submission. Please provide the name of the sponsor/funding agency below.

Trial Registration:

Trial registration : NCT02712892

Please provide the trial registration information for the study below. Include the trial registry, along with the unique identifying number. 
In Nantes, France

January 8, 2020

Dear Pr Nelson M. Oyesiku, Editor-in-Chief of Neurosurgery

In hopes of being published in your journal, we are pleased to offer you a proposal of our trial, entitled "Unruptured Cerebral Aneurysm, prediction of evolution: The UCAN Project"

Intracranial aneurysm rupture is a devastating event with a mortality rate as high as $40 \%$. Therefore, prediction and thus prevention of IA rupture is an attractive option to reduce the burden of this serious disease. Moreover, the management of small $(<7 \mathrm{~mm})$ unruptured intracranial aneurysms (UIA) remains controversial. Previous retrospective studies have suggested that post gadolinium arterial wall enhancement (AWE) of unruptured intracranial aneurysms on MR imaging may reflect aneurysm wall instability, and hence may highlight a higher risk of UIA growth over time. This multicentric prospective cohort aims at exploring vessel wall imaging findings of UIAs with consecutive follow-up to substantiate these assumptions.

We believe our trial is sufficiently novel, rigorous, and innovative to be considered for publication in Radiology, and therefore kindly submit our manuscript, "Unruptured Cerebral Aneurysm, prediction of evolution: The UCAN Project", for review. To write this article we followed SPIRIT recommendations

The UCAN Trial is a continuity of the ICAN project Bourcier et al (Neurosurgery, 2017). This first work gave results with the publication from Bourcier et al. (Rare Coding Variants in ANGPTL6 Are Associated with Familial Forms of Intracranial Aneurysm, in the American Journal of Human Genetics, 2018).

All authors have read and approved the submitted manuscript and no author has any related conflict of interest to disclose regarding this work. The manuscript has not been submitted elsewhere nor published elsewhere in whole or in part. 
Thank you very much for your kind consideration.

Regards

Dr. Vincent L'ALLINEC

Department of Neuroradiology - University Hospital of Nantes and Angers, France

Phone +33240165608-Fax +33240165616

Email: vincent.lallinec@gmail.com 


\section{Unruptured Cerebral Aneurysm, prediction of evolution: The UCAN Project}

Vincent L'Allinec, MD, MSc ${ }^{1,2,3}$; Stéphanie Chatel, $\mathrm{PhD}^{3}$; Matilde Karakachoff, $\mathrm{MSc}^{4}$; Emmanuelle Bourcereau, $\mathrm{BSc}^{5}$; Zeineb Lamoureux, $\mathrm{PhD}^{6}$; Alban Gaignard, $\mathrm{PhD}^{3}$; Florent Autrusseau, $\mathrm{PhD}^{7,8}$; Solène Jouan, $\mathrm{BSc}^{1}$; Anne-Clemence Vion, $\mathrm{PhD}^{3}$; Gervaise Loirand, $\mathrm{PhD}^{3}$; Hubert Desal, MD, $\mathrm{PhD}^{1,3}$; Olivier Naggara, $\mathrm{MD}, \mathrm{PhD}^{9}$; Richard Redon, $\mathrm{PhD}^{3}$; Myriam Edjlali, $\mathrm{MD}, \mathrm{PhD}^{* 9}$, Romain Bourcier, $\mathrm{MD}, \mathrm{PhD}^{* 1,3}$, on behalf of the UCAN (Unruptured Cerebral Aneurysm) Investigators.

*Myriam Edjlali and Romain Bourcier contributed equally to this work.

${ }^{1}$ CHU Nantes, Neuroradiology Department, Nantes, France

${ }^{2}$ Angers University Hospital, Radiology Department, Angers, France.

${ }^{3}$ Université de Nantes, CHU Nantes, CNRS, Inserm, l'institut du thorax, Nantes, France

${ }^{4}$ CHU Nantes, CIC Inserm 1413, Clinique des Données, Nantes, France

${ }^{5} \mathrm{CHU}$ Nantes, CIC Inserm 1413, l'institut du thorax, Nantes, France

${ }^{6} \mathrm{CHU}$ Nantes, Direction de la recherche, Nantes, France

${ }^{7}$ Université de Nantes, ONIRIS, Inserm UMR 1229, RMeS, Regenerative Medicine and

Skeleton, Nantes, France

${ }^{8}$ Ecole Polytechnique de l'Université de Nantes, Nantes, France

${ }^{9}$ Department of Neuroradiology, Université Paris-Descartes-Sorbonne-Paris-Cité, IMABRAIN-

INSERM-UMR1266, DHU-Neurovasc, Centre Hospitalier Sainte-Anne, Paris, France

\section{Correspondence:}

Vincent L'Allinec, MD, MSc,

Departement de Radiologie,

CHU Angers,

4 rue Larrey,

49000 Angers, France.

Email: $\underline{\text { Vincent.lallinec@gmail.com }}$

Disclosures: This study was supported by a grant from the French Ministry of Health (U-CAN, 2019, PHRC-19-0394). The authors have no personal, financial, or institutional interest in any of the drugs, materials, or devices described in this article.

Acknowledgments: We are also grateful to the UCAN collaborators, composed of Bourcier R, Daumas-Duport B, Lenoble C, Alexandre PL, Detraz L, Pellerin A, Lintia-Gaultier A, AuffrayCalvier E, Aguilar J, Desal H (University Hospital of Nantes); Pasco A, L'Allinec V, Tanguy JY, Labriffe M, Girot JB (University Hospital of Angers); Anxionnat R, Tonnelet R, Derelle AL, Barbier C, Bracard S, Gory B (University Hospital of Nancy); Ferré JC, Raoult H, Eugène F, Gauvrit JY, Paya C (University Hospital of Rennes); Gentric JC, Ognard J, Douraied B (University Hospital of Brest); Chivot C (University Hospital of Amiens); Papagiannaki C, Gerardin E (University Hospital of Rouen); Januel AC, Bonneville F, Cognard C, Michelozzi C, Darcourt J (University Hospital of Toulouse); Levrier O, Aguettaz P (Hospital of Clairval, Marseille); Naggara O, Trystram D, Rodriguez-Regent C, Ben Hassen W, Boulouis G, Meder JF (Saint Anne Hospital-Paris); Rouchaud A, Mounayer C, Saleme S (University Hospital of 
Limoges); Houdart E, Labeyrie MA (University Hospital of Lariboisière AP-HP); Pierot L, Soize S (University hospital of Reims); Gariel F, Barreau X, Berge J, Marnat G (University hospital of Bordeaux); Caroff G, Spelle L, Ozanne A (University hospital of Kremlin Bicêtre, AP-HP);

Shotar E, Clarencon F (University Hospital of Pitié Salpétriere, AP-HP); Herbreteau D, Janot K, Cottier JP (University hospital of Tours); Desilles JP, Blanc R, Piotin M (Fondation Roschild Paris)
Commented [SJ1]: Typesetter : These names should all be listed in PubMed as non-author collaborators 


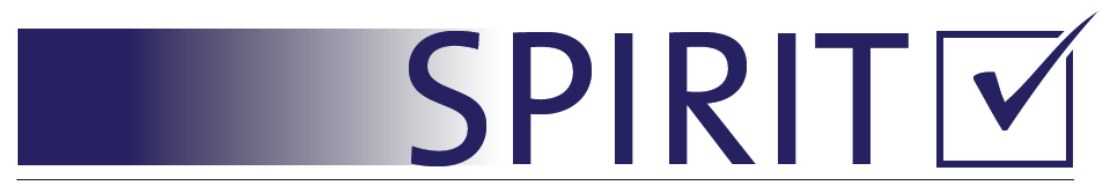

Standard Protocol Items: Recommendations for INTERVEntional Trials

SPIRIT 2013 Checklist: Recommended items to address in a clinical trial protocol and related documents*

\section{Section/item Item Description \\ No}

\section{Administrative information}

Title

Trial registration

Protocol version

Funding

Roles and responsibilities
1 Descriptive title identifying the study design, population, interventions, and, if applicable, trial acronym $\checkmark$ Page 1

2a Trial identifier and registry name. If not yet registered, name of intended registry $\sqrt{ }$ Page 1

2b All items from the World Health Organization Trial Registration Data Set

3 Date and version identifier $\sqrt{ }$ Page 1

4 Sources and types of financial, material, and other support $\sqrt{ }$ Page 1

5a Names, affiliations, and roles of protocol contributors

5b Name and contact information for the trial sponsor Page 1, 10, 13 and 14

5c Role of study sponsor and funders, if any, in study design; collection, management, analysis, and interpretation of data; writing of the report; and the decision to submit the report for publication, including whether they will have ultimate authority over any of these activities

$5 d$ Composition, roles, and responsibilities of the coordinating centre, steering committee, endpoint adjudication committee, data management team, and other individuals or groups overseeing the trial, if applicable (see Item 21a for data monitoring committee)

\section{Introduction}

Background and

6a Description of research question and justification for undertaking the rationale trial, including summary of relevant studies (published and unpublished) examining benefits and harms for each intervention

6b Explanation for choice of comparators

Objectives

7 Specific objectives or hypotheses Page 4

Trial design

8 Description of trial design including type of trial (eg, parallel group, crossover, factorial, single group), allocation ratio, and framework (eg, superiority, equivalence, noninferiority, exploratory) 


\section{Methods: Participants, interventions, and outcomes}

Study setting 9 Description of study settings (eg, community clinic, academic hospital) and list of countries where data will be collected. Reference to where list of study sites can be obtained $\checkmark$ Page 4 and 13

Eligibility criteria 10 Inclusion and exclusion criteria for participants. If applicable, eligibility criteria for study centres and individuals who will perform the interventions (eg, surgeons, psychotherapists) $\sqrt{ }$ Page 5 and 6

Interventions $\quad 11 \mathrm{a}$ Interventions for each group with sufficient detail to allow replication, Page 10

11b Criteria for discontinuing or modifying allocated interventions for a given trial participant (eg, drug dose change in response to harms, participant request, or improving/worsening disease)

11c Strategies to improve adherence to intervention protocols, and any procedures for monitoring adherence (eg, drug tablet return, laboratory tests)

11d Relevant concomitant care and interventions that are permitted or prohibited during the trial

Outcomes

Page 4 and 5

Participant timeline

Page 10

Sample size

Page 7

Recruitment

Page 5
12

Primary, secondary, and other outcomes, including the specific measurement variable (eg, systolic blood pressure), analysis metric (eg, change from baseline, final value, time to event), method of aggregation (eg, median, proportion), and time point for each outcome. Explanation of the clinical relevance of chosen efficacy and harm outcomes is strongly recommended

13 Time schedule of enrolment, interventions (including any run-ins and washouts), assessments, and visits for participants. A schematic diagram is highly recommended (see Figure)

14 Estimated number of participants needed to achieve study objectives and how it was determined, including clinical and statistical assumptions supporting any sample size calculations

15 Strategies for achieving adequate participant enrolment to reach target sample size

\section{Methods: Assignment of interventions (for controlled trials)}

Allocation:

Sequence generation 16a Method of generating the allocation sequence (eg, computergenerated random numbers), and list of any factors for stratification. To reduce predictability of a random sequence, details of any planned restriction (eg, blocking) should be provided in a separate document that is unavailable to those who enrol participants or assign interventions 
Allocation concealment mechanism

Implementation $16 \mathrm{c}$

Blinding (masking) 16b Mechanism of implementing the allocation sequence (eg, central telephone; sequentially numbered, opaque, sealed envelopes), describing any steps to conceal the sequence until interventions are assigned

Who will generate the allocation sequence, who will enrol participants, and who will assign participants to interventions

17a Who will be blinded after assignment to interventions (eg, trial participants, care providers, outcome assessors, data analysts), and how

17b If blinded, circumstances under which unblinding is permissible, and procedure for revealing a participant's allocated intervention during the trial

\section{Methods: Data collection, management, and analysis Page 6}

Data collection methods

Data management

Statistical methods 18a Plans for assessment and collection of outcome, baseline, and other trial data, including any related processes to promote data quality (eg, duplicate measurements, training of assessors) and a description of study instruments (eg, questionnaires, laboratory tests) along with their reliability and validity, if known. Reference to where data collection forms can be found, if not in the protocol

18b Plans to promote participant retention and complete follow-up, including list of any outcome data to be collected for participants who discontinue or deviate from intervention protocols

19 Plans for data entry, coding, security, and storage, including any related processes to promote data quality (eg, double data entry; range checks for data values). Reference to where details of data management procedures can be found, if not in the protocol

20a Statistical methods for analysing primary and secondary outcomes. Reference to where other details of the statistical analysis plan can be found, if not in the protocol

20b Methods for any additional analyses (eg, subgroup and adjusted analyses)

20c Definition of analysis population relating to protocol non-adherence (eg, as randomised analysis), and any statistical methods to handle missing data (eg, multiple imputation)

\section{Methods: Monitoring $\sqrt{ }$ Page 6}

Data monitoring 21a Composition of data monitoring committee (DMC); summary of its role and reporting structure; statement of whether it is independent from the sponsor and competing interests; and reference to where further details about its charter can be found, if not in the protocol. Alternatively, an explanation of why a DMC is not needed 
21b Description of any interim analyses and stopping guidelines, including who will have access to these interim results and make the final decision to terminate the trial

Harms 22 Plans for collecting, assessing, reporting, and managing solicited and spontaneously reported adverse events and other unintended effects of trial interventions or trial conduct

Auditing 23 Frequency and procedures for auditing trial conduct, if any, and whether the process will be independent from investigators and the sponsor

\section{Ethics and dissemination $\sqrt{ }$ Page 9}

Research ethics 24 Plans for seeking research ethics committee/institutional review board approval (REC/IRB) approval

Protocol amendments

Consent or assent $26 \mathrm{a}$

$26 b$

Confidentiality

27

Declaration of interests

Access to data

Ancillary and post-trial care

Dissemination policy
25 Plans for communicating important protocol modifications (eg, changes to eligibility criteria, outcomes, analyses) to relevant parties (eg, investigators, REC/IRBs, trial participants, trial registries, journals, regulators)

Who will obtain informed consent or assent from potential trial participants or authorised surrogates, and how (see Item 32)

Additional consent provisions for collection and use of participant data and biological specimens in ancillary studies, if applicable

27 How personal information about potential and enrolled participants will be collected, shared, and maintained in order to protect confidentiality before, during, and after the trial

28 Financial and other competing interests for principal investigators for the overall trial and each study site

29 Statement of who will have access to the final trial dataset, and disclosure of contractual agreements that limit such access for investigators

30 Provisions, if any, for ancillary and post-trial care, and for compensation to those who suffer harm from trial participation

31a Plans for investigators and sponsor to communicate trial results to participants, healthcare professionals, the public, and other relevant groups (eg, via publication, reporting in results databases, or other data sharing arrangements), including any publication restrictions

31b Authorship eligibility guidelines and any intended use of professional writers

31c Plans, if any, for granting public access to the full protocol, participantlevel dataset, and statistical code 


\section{Appendices}

Informed consent 32 Model consent form and other related documentation given to materials participants and authorised surrogates

Biological

33 Plans for collection, laboratory evaluation, and storage of biological specimens specimens for genetic or molecular analysis in the current trial and for Page 6 future use in ancillary studies, if applicable

${ }^{*}$ It is strongly recommended that this checklist be read in conjunction with the SPIRIT 2013 Explanation \& Elaboration for important clarification on the items. Amendments to the protocol should be tracked and dated. The SPIRIT checklist is copyrighted by the SPIRIT Group under the Creative Commons "Attribution-NonCommercial-NoDerivs 3.0 Unported" license. 


\section{ABSTRACT}

Background: Management of small $(<7 \mathrm{~mm})$ unruptured intracranial aneurysms (UIA) remains controversial. Retrospective studies have suggested that post gadolinium arterial wall enhancement (AWE) of UIA on MRI may reflect aneurysm wall instability, and hence may highlight a higher risk of UIA growth. This trial aims at exploring wall imaging findings of UIAs with consecutive follow-up to substantiate these assumptions.

Objective: To develop diagnostic and predictive tools for the risk of IA evolution. Our aim is to demonstrate in clinical practice the predictive value of AWE for UIA growth. The growth will be determined by any modification of the UIA measurement. UIA growth and the UIA wall enhancement will be assessed in consensus by two expert neuroradiologists.

Methods: The French prospective UCAN project is a non-interventional international wide and multicentric cohort. UIA of bifurcation between 3 and $7 \mathrm{~mm}$ for whom a clinical and imaging follow-up without occlusion treatment was scheduled by local multidisciplinary staff will be included. Extensive clinical, biological and imaging data will be recorded during a 3 years follow-up.

Expected Outcomes: Discovering to improve the efficiency of UIA follow-up by identifying additional clinical, imaging, biological and anatomical risk factors of UIAs growth.

Discussion: A prospective nationwide recruitment allows for the inclusion of a large cohort of patients with UIA. It will combine clinical phenotyping and specific imaging with AWE screening. It will enable to exploit metadata and to explore some pathophysiological pathways by crossing clinical, genetic, biological, and imaging information.

Keywords: aneurysm, trial, MRI, growth, Intracranial aneurysm, wall enhancement, CAWE Abbreviations: UIA (unruptured intracranial aneurysm); AWE (arterial wall enhancement); CAWE (circumferential arterial wall enhancement)

\section{GENERAL INFORMATION}

\section{Study Dates}

Recruitment phase: August 2019 to august 2021, this study is ongoing Follow-up phase: August 2020 to April 2024 


\section{Funding Agencies}

This study was supported by a grant from the French Ministry of Health (U-CAN, 2019, PHRC19-0394)

\section{Registry}

(Clinical Trial) NCT02712892

\section{Investigators}

The project management is ensured by:

- Dr Romain Bourcier, MD, PhD, from the department of Neuroradiology of the Nantes University Hospital, Bd Jacques Monod, St Herblain, 44093 NANTES Cedex 1, France

- Dr Myriam Edjlali, MD, PhD, From the department of Neuroradiology, Sainte-Anne Hospital, Paris Descartes University, 1 rue Cabanis, Paris 14, Paris, France

Trial sponsor:

Zeineb Lamoureaux, zeineb.lamoureux@chu-nantes.fr

CHU Nantes, Direction de la recherche, 44093 Nantes, France

\section{RATIONALE AND BACKGROUD INFORMATION}

The overall prevalence of unruptured IA (UIA) is estimated to be between $2 \%$ and $4 \%$ in the general population ${ }^{1,2}$. IA rupture is a devastating event with a mortality rate as high as $40 \%{ }^{3}$. Therefore, prediction and thus prevention of IA rupture is an interesting way of research to reduce the impact of this serious complication. Currently, the management or the follow-up for asymptomatic UIA is still based on the benefit-risk balance without clear guidelines ${ }^{4-6}$.

According to the natural history model for IA proposed by Yonekura et al, ${ }^{7}$ IA may remain stable for a long time after formation, may form and grow before rupture or may rupture immediately after aneurysm formation. Consequently, the growth of IA could be used as an imaging marker and may predict the risk of rupture. A systematic review from Gondar et $\mathrm{al}^{8}$ with 
the follow up of 3855 patients identified an estimation of a yearly growth probability of $3.85 \%$ $(95 \% \text { CI } 3.4 \% \text { to } 4.3 \%)^{8}$. Hence, follow-up imaging of untreated UIAs is recommended, several studies having suggested that growing UIAs have an increased risk of rupture ${ }^{9-12}$. According to Inoue et $\mathrm{al}^{13}$, and Villablanca et $\mathrm{al}^{12}$, UIA growth increases the risk of rupture by a factor 10 and the annual rupture rate for a growing UIA ranges between $2.4 \%$ and $18 \%$ per patient-year. Furthermore, in a meta-analysis with 4990 IAs, the annual risk of rupture was associated with over a 30-fold higher when an UIAs were growing than stable ${ }^{14}$. However, guidelines from the American Heart Association and European Stroke Organization lack recommendations on which patients' follow-up imaging should be considered for and at what time interval it should be performed ${ }^{9,15}$.

In this context, the identification of a specific individual-based marker for higher risk of IA growth can help to the therapeutic choice. A performant imaging marker for UIA instability would permit physicians to choose between conservative management or requiring invasive treatment to prevent rupture.

Preliminary studies have demonstrated that aneurysmal wall enhancement (AWE), using highresolution vessel wall MR imaging, is linked to IA instability (i.e., ruptured, symptomatic, or growing over time). Indeed, some studies that included ruptured IAs and UIAs studied by a 3.0-T MRI with vessel wall sequences, suggest that CAWE was more frequently present in unstable rather than in stable IAs ${ }^{16-19}$. In a study focused on a large monocentric cohort of more than 300 UIAs, it was demonstrated that strong CAWE was a more specific marker to discriminate stable from unstable UIAs (specificity $84.4 \%)^{20}$.

\section{STUDY GOALS AND OBJECTIVES}

In order to bring to light specific biomarkers of instability, a prospective multicentric study warrants the demonstration in clinical practice of the predictive value of AWE for UIA growth. Such an investigation will allow to set up a secure, efficient and personalized follow-up for each IA. Having an individual-based imaging marker for UIA instability would permit physicians to 
characterize and discriminate IA as appropriate for conservative management or as requiring invasive treatment to prevent rupture.

\section{STUDY DESIGN}

The French prospective UCAN project is a non-interventional international wide and multicentric prospective cohort

\section{Objectives and Endpoints}

\section{Primary Objective}

Our aim is to evaluate in clinical practice the predictive value of UIA wall enhancement for UIA growth. It will allow setting up a secure, efficient and personalized follow-up.

\section{Primary Endpoint}

In order to evaluate the informative value of AWE for UIA growth, we will consider as primary endpoint the growth of the UIA after the complete follow-up at 3 years. This event could occur at any time during the follow-up if an UIA becomes symptomatic but will be systematically assessed at 1 year and 3 years by MRI. UIA growth will be assessed blindly and independently by two expert neuroradiologists, routinely involved in UIA management and disagreement will be solved by consensus with involvement of a third expert. UIA wall enhancement status will be defined independently by two different expert neuroradiologists, with > 5yrs experience in intracranial vessel wall imaging. Disagreement will be solved by consensus with involvement of a third expert.

\section{Secondary Objectives}

- Determination of clinical, genetics or biological factors related to the growth of UIA.

- Determination of clinical, genetics or biological factors related to rupture of UIA.

- $\quad$ Evaluation of the quality of life (QOL) of untreated patients with UIA during the follow-up. 
- $\quad$ Detection of other AWE variation patterns related to growth during the follow-up in order to improve the follow-up of UIA patients.

\section{Secondary Endpoints}

- $\quad$ Clinical, genetic (blood serum level of circulating ANGPTL6) and biological (plasma factors as circulating ANGPTL6 levels, metalloproteinase...) features recorded.

- $\quad$ incidence of growth, stratified by clinical, genetics or biological features.

- $\quad$ incidence of IA rupture, stratified by clinical, genetics or biological features.

- $\quad$ Completion of standardized EQ5D questionnaire to measure quality of life patients (50)

- Construction and evaluation of an automatized tool of AWE patterns, as compared to the visual analysis of experts, in the form of a decision-making tool.

\section{Definition of UIA and AWE}

A typical UIA is a saccular arterial dilatation localized at a intracranial bifurcation. The phenotyping of UIA is performed in each center by experienced interventional neuroradiologists, neurologists, and neurosurgeons to exclude other IA types (i.e. fusiform-shaped, dissection) and to include only typical UIA.

The definition of AWE is broad, encompasses both thin and thick, partial or circumferential enhancements, and does not capture focal eccentric enhancement. In a recent study focused on patients with UIA, a thick (>1 mm) circumferential pattern of wall enhancement demonstrated the highest specificity for differentiating between stable and unstable UIA ${ }^{20}$. In this study, the presence of AWE will be graded with the classification proposed by Edjlali and al $^{20}$.

\section{Inclusion and Exclusion Criteria}

The recruited population is composed of subjects carrying unruptured asymptomatic typical IA of bifurcation for whom a clinical, a biological and an imaging follow-up, without occlusion treatment, was scheduled by local multidisciplinary staff. 


\section{Inclusion Criteria}

- Subject carrying unruptured, asymptomatic and untreated typical IA of bifurcation, measured on conventional imaging (MRI, CTA or DSA) between 3 and $7 \mathrm{~mm}$ of larger diameter.

- Ability to be followed-up during 3 years decided in consensus multidisciplinary gathering.

- Age > 18 years old.

- UIA discovery less than 24 months ago.

\section{Non-inclusion Criteria}

- A failure to obtain informed consent.

- Contraindications for undergoing an MRI scan include: (heart pacemaker, a metallic foreign body (metal sliver) in their eye, or aneurysm clip in their brain, severe claustrophobia)

- Contraindications for a gadolinium contrast medium injection (:eGFR below 30 $\mathrm{mL} / \mathrm{min} / 1.73 \mathrm{~m} 2$, Previous or pre-existing nephrogenic systemic fibrosis, Previous anaphylactic/anaphylactoid reaction to gadolinium containing contrast agent, Acutely deteriorating renal function, Pregnancy and breast-feeding)

- A fusiform-shaped, mycotic, an IA in relation with an arteriovenous malformation dissecting IA

- A family history of Ehlers-Danlos syndrome, polycystic kidney disease, fibromuscular dysplasia, Marfan's syndrom, or Moya Moya disease.

- Intra-cavernous UIA because the sinus cavernous that is fulfilled with venous blood precluded a reliable assessment of aneurysmal wall enhancement (AWE).

- Partially or completely thrombosed UIA because of the inherent enhancement of the parietal layer in that case.

\section{METHODOLOGY}


This study presents the following characteristics: international, multicentric and prospective cohort

\section{Data Recorded}

\section{Clinical Data}

Data related to environmental risk factors are collected for each included patient like smoking history, parameters as high blood pressure, diabetes mellitus, hyper-cholesterolemia, alcohol consumption, body mass index. In order to better interpret the apparition of the contrast enhancement of the UIA, a very precise interview will be carried out to unravel recent infections, dental care or any other acute as well as chronical or recent event that can lead to inflammatory reactions. In case of multi-aneurysm in one patient, if one aneurysm is treated during the study, follow-up of other untreated aneurysms will be maintained. If all aneurysms of one patient are treated during the study, research follow-up will be suspended. Information about treatment will be collected. Moreover the standardized EQ5D questionnaire will be administrated in order to measure the impact of follow-up on patient's quality of life.

\section{Biological Data}

The biocollection will consist of blood sampling; $10 \mathrm{ml}$ for DNA analysis during the inclusion (V0), $10 \mathrm{ml}$ of plasma at each visit (V0, V1 at 1 year and V2 at 3 years).

\section{Imaging Data}

Sequences will be made coherent for each center and each MRI, to optimize the evaluation of AWE. Imaging the vessel wall implicates a 3T MRI with specific 3D T1 fast spin echo sequence, realized before and after gadolinium injection. Hence, the spatial resolution is crucial for the analysis of such a small vessel part, and every participating center will have to obtain a 3D T1 fast spin echo sequence with a spatial resolution of at least $0.9 \times 0.9 \times 1 \mathrm{~mm}$. Such sequence usually lasts 5 minutes. The optimal protocol will follow these parameters: field of view, $23 \times 23 \times 16 \mathrm{~cm}$ 3; repetition time/echo time, $600 / 11.5 \mathrm{~ms}$; spatial resolution: $0.45 \times 0.45 \times 0.5 \mathrm{~mm}$; matrix, $288 \times 288 \times 160$ interpolated to $512 \times 512 \times 320)$. 


\section{DISCUSSION}

With the UCAN project and using a combination of innovative aspects in terms of approach, interdisciplinary collaboration and technologies, we aim to improve the prediction of IA growth by identifying advanced but routinely accessible imaging parameters as well as clinical, anatomical and biological risk factors for IA growth. This project establishes new directions for optimal and personalized management of UIAs to decrease the impact of futile follow-up and the risk of unrecognized, evolving UIA. Overall, we could also expect to improve the impact of follow-up on the patient's quality of life.

\section{TRIAL STATUS}

This is an ongoing study.

\section{SAFETY CONSIDERATIONS}

No adverse event or reaction can be associated with this study as it consists on a noninterventional study with no impact on care. The onset of an Adverse Reaction associated with patient care in the course of this protocol shall be reported in the suitable vigilance system (pharmacovigilance, biovigilance, hemovigilance, medical device vigilance, etc.).

\section{FOLLOW-UP}

3 visits are planned:

Visit 0: MRI with contrast injection, Blood sampling for serum, plasma and genetics Visit 1: 1 year after inclusion, MRI with contrast injection, blood sampling for serum and plasma

Visit 2: 3 years after inclusion, MRI with contrast injection, blood sampling for serum and plasma

\section{DATA MANAGEMENT AND STATISTICAL ANALYSIS}


This is a prospective, multicenter cohort study with 1000 patients.

A review of the data will be conducted at the end of the study, before the statistical analysis. During this review the following will be present: principal investigator, project manager, CRA monitoring, data manager, a statistician and anyone concerned with the protocol. The aim will be to review the progress of the study on the possible problems and classify possible deviations in minor or major.

Demographic, clinical, IRM, genetics or biological data will be recorded per patient. For categorical and binary variables, counts and percentages will be calculated. For continuous variables, means, standard deviations, the minimum and maximum, medians and interquartile range will be calculated. For binary variables such as gender, counts, percentages, and 95\% confidence intervals will be calculated, and p-values may be presented for hypothesis generating purposes. Pearson's Chi-squared test or Fisher's exact test will be performed when appropriate. The Mann-Whitney-U and t- tests will be performed to test for statistical differences in continuous parameters (based on distribution and count). For patients lost to follow-up, the status of the last follow-up examination will be recorded. Analyses will be performed using the R statistics software (R Foundation for Statistical Computing, Vienna, Austria) through the Rstudio interface (RStudio, Inc., Boston, Massachusetts)

No interim analysis is planned.

\section{Principal Analysis on Primary Endpoint}

Our aim is to demonstrate in clinical practice the predictive value of UIA wall enhancement for UIA growth. The growth will be determined by any modification of the UIA measurement at the end of the follow-up and categorized as present/absent. UIA wall enhancement will be assessed in consensus by two expert neuroradiologists and categorized as present/absent. To demonstrate the predictive value of AWE for UIA growth we will measure the ability of AWE to discriminate between stable and unstable UIA through a diagnostic accuracy study. We will compute sensitivity, specificity, accuracy, negative and positive predictive values for presence/absence of AWE to identify unstable status. Related 95\% CI for each measure will be 
calculated using the binomial Clopper-Pearson method (ref 51). The association between AWE and UIA growth will be tested through a Pearson's chi-squared test or Cochran Mantel Haeszel test, if stratification is necessary. Montecarlo simulation method will be used for estimating pvalues.

Intra and inter reader agreement for presence/absence for AWE and, separately, for growing status will be assessed calculating intra/inter class correlation by Cohen $\mathrm{k}$.

\section{Analysis of Secondary Endpoints}

Determination of clinical, genetics or biological factors related to the growth of UIA.

Conditional univariate logistic regression models will be applied to explore the putative association between UIA growth and patient clinical, genetic and biological features. A multiple logistic regression model will be thus applied to identify patient characteristics and assess their combined effect on UIA growth. Age and sex will be considered as confounder variables.

Determination of clinical, genetics or biological factors related to rupture of UIA.

The same methods will be used also to identify patients characteristics related to rupture of UIA.

Evaluation of the quality of life (QOL) of untreated patients with UIA during the follow-up. Responses to EQ5D questionnaire will be analyzed in order to determine the evolution of patient's quality of life during the follow-up. Differences will be measured between two different intervals of follow-up. Detection of other AWE variation patterns related to growth during the follow-up. Based on the large sample size, the prospective and standardized follow-up as well as the number of accurate variables registered in UCAN, we will integrate the whole of the recorded anatomical and clinical data in order to enrich the predicting strategy in the form of a risk score. The large number of variables will be used in order to identify combinations of markers that can help with predicting growth (and ultimately rupture). If such (most likely nonlinear) combinations of variables exist, they can be a great help in managing UIA prevention. Given the large number of variables and the variability in distribution of their shapes (normal, multi-modal, categorical etc.), we will adopt decision methods such as multi-dimensional reduction methods or regression trees. As a result, we hope to identify a subset of variables - 
possibly assembled into a score - which can determine AWE patterns and thus predict the UIA growth.

\section{Statistical Justification of the Number of Inclusions}

We know from precedent studies ${ }^{20}$ that $17 \%$ of individuals with AWE manifest an unstable UIA. Among individuals without AWE, the proportion of unstable UIA is 5\%. A sample size of almost 300 patients is needed to show a significant difference of the proportion of unstable UIA between AWE and not AWE patients ( $\beta=90 \%$ and $\alpha=5 \%$ ). From the same study, we know that any UIA wall enhancement was seen in $42 \%$ and UIA instability was encountered in $10 \%$ of UIA patients. To have enough unstable UIA to make multivariate analysis, we can expect more than 50 evolutions among UIA. With 1000 patients, we can expect between 50 and 75 evolutions and 420-450 apparitions of any enhancement.

Moreover, the current data from the literature give estimations of the specificity of AWE to discriminate between unstable and stable UIA comprised between 60 and 85\% (39). In our selected population we expect this proportion, i.e. the proportion of negative AWE among not growing UIA, to be at least the same after three years follow-up. With an alpha risk set to 0.05 and an expected specificity of $60 \%$, a population size of 1000 patients will give us a total width of confidence interval of 6\% [95\%CI 56.9-63.1\%], using binomial (Clopper-Pearson) "exact" calculation (see Table, Supplemental Digital Content for a summary of $95 \% \mathrm{CI}$ according to different scenarios, supplementary data).

The expected level of statistical significance is $5 \%$.

The image analysis is ensured by:

- Florent Autrusseau, PhD, from Inserm, UMR 1229, RMeS, Regenerative Medicine and Skeleton, 1, place A. Ricordeau, Nantes University, ONIRIS, Nantes, F-44042, France. Dr Autrusseau will specifically be involved in the study of the evolution of the CAWE from a digital image analysis perspective. 


\section{QUALITY ASSURANCE}

A monitoring shall be carried out by the Research Division Promotion Department. A Clinical Research Associate will remotely conduct quality control on the data reported in the case report forms. The monitoring plan is defined in close cooperation between the study team and the responsible institution according to the objectives of the study, based on an internal Research Division procedure.

The on-site monitoring visits shall be organized after making arrangements with the investigator. The Clinical Research Associate should be able to consult on each site:

- the enrolled patients' data compilation records,

- the patients' medical and nursing files,

- the investigator file.

Within the scope of this study, an inspection or audit may be conducted. The sponsor and/or participating centers should be able to provide inspectors or auditors with access to the data.

\section{EXPECTED OUTCOMES OF THE STUDY}

We expect to improve the UIA follow-up and management by identifying additional clinical, anatomical, biological and imaging factors of UIAs growth. This project is designed to establish new individual biomarkers including imaging biomarkers for optimal early recognition of UIAs prone to evolve, in order to improve UIA management, and will use a combination of innovative aspects in terms of approach, interdisciplinary collaboration and technology. It will provide novel tools for identification of high-risk individuals and for improving efficiency of UIA follow-up. The gain in knowledge on additional risk factors will also pave the way for novel, non-invasive treatment strategies and timing of treatment in future clinical practice.

\section{DURATION OF THE PROJECT}

The period of the study will be 5 years. We have scheduled a recruitment period of 2 years (August 2019 to August 2021) and a follow-up period of 3 years. 


\section{PROJECT MANAGEMENT}

The project management is ensured by:

- Dr Romain Bourcier, MD, PhD form the Neuroradiology Department, Nantes University Hospital, 8 Quai Moncousu, 44000 Nantes, France

- Dr Myriam Edjlali, MD, PhD, from the Department of Neuroradiology, Université ParisDescartes-Sorbonne-Paris-Cité, IMABRAIN-INSERM-UMR1266, DHU-Neurovasc, Centre Hospitalier Sainte-Anne, Paris, France.

The imaging management is ensured by:

- Dr Myriam Edjlali, MD, PhD, from the Department of Neuroradiology, Université ParisDescartes-Sorbonne-Paris-Cité, IMABRAIN-INSERM-UMR1266, DHU-Neurovasc, Centre Hospitalier Sainte-Anne, Paris, France

The statistical management is ensured by:

- Mathilde Karakachoff, Clinique des Données CIC Inserm 1413, CHU Nantes, 30 boulevard Jean Monnet

The biological management is ensured by:

- Dr Richard Redon, L'institut du thorax Nantes, INSERM, CNRS, UNIV Nantes, Centre Hospitalier Universitaire Nantes, Nantes, France.

The image analysis is ensured by:

- Florent Autrusseau, PhD from Inserm, UMR 1229, RMeS, Regenerative Medicine and Skeleton, 1, place A. Ricordeau, Nantes University, ONIRIS, Nantes, F-44042, France. F. Autrusseau will specifically be involved in the study of the evolution of the CAWE from a digital image analysis perspective.

\section{ETHICS}

\section{Patient Information}

The investigator undertakes to inform the patient of the protocol in clear and accurate terms (information form appended). The investigator shall provide the patient with a copy of the information form. This document shall specify to the patient that he/she has the option of refusing to take part in the study and can withdraw at any time. 


\section{Ethical Review Board}

The sponsor commits to submit the study project to the prior authorization of an ethical review board (ERB). The information sent concern on one hand the modalities and the nature of the research, and, on the other hand, the guarantees planned for the persons taking part in the study.

\section{Source Data and Document Access Rights}

Each patient's medical data shall only be provided to the affiliated body of the study director or any person duly authorized thereby, in confidential conditions. If applicable, the affiliated body of the director may request direct access to the medical file for the purposes of verification of the procedures and/or data in respect of the clinical trial, without breaching confidentiality and within the limits authorized by the legislation and regulations.

Computerized data and submission to the French data protection authority (CNIL) The data gathered during the study shall be held on computerized file, as per the 2004 amendment of the French data protection act of 6 January 1978, the law $n^{\circ} 2018-493$ of June 20th 2018 on the Protection of Personal Data and Regulation (EU) 2016/679 of the European Parliament and of the Council of 27 April 2016 on the protection of individuals with regard to the processing of personal data and on the free movement of such data (General Data Protection Regulation). The protocol falls within the scope of the MR004 methodology applied by.

\section{REFERENCES}

1. Rinkel GJ, Djibuti M, Algra A, van Gijn J. Prevalence and risk of rupture of intracranial aneurysms: a systematic review. Stroke. 1998;29(1):251-256.

2. Vlak MH, Algra A, Brandenburg R, Rinkel GJ. Prevalence of unruptured intracranial aneurysms, with emphasis on sex, age, comorbidity, country, and time period: a systematic review and meta-analysis. Lancet Neurol. 2011;10(7):626-636. doi:10.1016/S14744422(11)70109-0 
3. Nieuwkamp DJ, Setz LE, Algra A, Linn FHH, de Rooij NK, Rinkel GJE. Changes in case fatality of aneurysmal subarachnoid haemorrhage over time, according to age, sex, and region: a meta-analysis. Lancet Neurol. 2009;8(7):635-642. doi:10.1016/S14744422(09)70126-7

4. Wiebers DO, Whisnant JP, Huston J, et al. Unruptured intracranial aneurysms: natural history, clinical outcome, and risks of surgical and endovascular treatment. Lancet Lond Engl. 2003;362(9378):103-110. doi:10.1016/s0140-6736(03)13860-3

5. Naggara O, Darsaut T, Trystram D, Tselikas L, Raymond J. Unruptured intracranial aneurysms: why we must not perpetuate the impasse for another 25 years. Lancet Neurol. 2014;13(6):537-538. doi:10.1016/S1474-4422(14)70091-2

6. Naggara ON, Lecler A, Oppenheim C, Meder J-F, Raymond J. Endovascular treatment of intracranial unruptured aneurysms: a systematic review of the literature on safety with emphasis on subgroup analyses. Radiology. 2012;263(3):828-835. doi:10.1148/radiol.12112114

7. Yonekura M, Sakurai Y, Kikuchi H. [Natural history and annual rupture rate on unruptured intracranial aneurysm]. Nihon Rinsho Jpn J Clin Med. 2006;64 Suppl 8:614-618.

8. Gondar R, Gautschi OP, Cuony J, et al. Unruptured intracranial aneurysm follow-up and treatment after morphological change is safe: observational study and systematic review. $J$ Neurol Neurosurg Psychiatry. 2016;87(12):1277-1282. doi:10.1136/jnnp-2016-313584

9. Thompson BG, Brown RD, Amin-Hanjani S, et al. Guidelines for the Management of Patients With Unruptured Intracranial Aneurysms: A Guideline for Healthcare Professionals From the American Heart Association/American Stroke Association. Stroke. 2015;46(8):2368-2400. doi:10.1161/STR.0000000000000070

10. Mehan WA, Romero JM, Hirsch JA, et al. Unruptured intracranial aneurysms conservatively followed with serial CT angiography: could morphology and growth predict rupture? J Neurointerventional Surg. 2014;6(10):761-766. doi:10.1136/neurintsurg-2013010944 
11. Chmayssani M, Rebeiz JG, Rebeiz TJ, Batjer HH, Bendok BR. Relationship of growth to aneurysm rupture in asymptomatic aneurysms $\leq 7 \mathrm{~mm}$ : a systematic analysis of the literature. Neurosurgery. 2011;68(5):1164-1171; discussion 1171. doi:10.1227/NEU.0b013e31820edbd3

12. Villablanca JP, Duckwiler GR, Jahan R, et al. Natural history of asymptomatic unruptured cerebral aneurysms evaluated at CT angiography: growth and rupture incidence and correlation with epidemiologic risk factors. Radiology. 2013;269(1):258-265. doi:10.1148/radiol.13121188

13. Inoue T, Shimizu H, Fujimura M, Saito A, Tominaga T. Annual rupture risk of growing unruptured cerebral aneurysms detected by magnetic resonance angiography. J Neurosurg. 2012;117(1):20-25. doi:10.3171/2012.4.JNS112225

14. Brinjikji W, Zhu Y-Q, Lanzino G, et al. Risk Factors for Growth of Intracranial Aneurysms: A Systematic Review and Meta-Analysis. AJNR Am J Neuroradiol. 2016;37(4):615-620. doi:10.3174/ajnr.A4575

15. Steiner T, Juvela S, Unterberg A, et al. European Stroke Organization guidelines for the management of intracranial aneurysms and subarachnoid haemorrhage. Cerebrovasc Dis Basel Switz. 2013;35(2):93-112. doi:10.1159/000346087

16. Edjlali M, Gentric J-C, Régent-Rodriguez C, et al. Does aneurysmal wall enhancement on vessel wall MRI help to distinguish stable from unstable intracranial aneurysms? Stroke. 2014;45(12):3704-3706. doi:10.1161/STROKEAHA.114.006626

17. Matouk CC, Mandell DM, Günel M, et al. Vessel wall magnetic resonance imaging identifies the site of rupture in patients with multiple intracranial aneurysms: proof of principle. Neurosurgery. 2013;72(3):492-496; discussion 496. doi:10.1227/NEU.0b013e31827d1012

18. Fu Q, Guan S, Liu C, Wang K, Cheng J. Clinical Significance of Circumferential Aneurysmal Wall Enhancement in Symptomatic Patients with Unruptured Intracranial 
Aneurysms: a High-resolution MRI Study. Clin Neuroradiol. 2018;28(4):509-514.

doi:10.1007/s00062-017-0598-4

19. Omodaka S, Endo H, Niizuma K, et al. Circumferential Wall Enhancement on Magnetic Resonance Imaging is Useful to Identify Rupture Site in Patients with Multiple Cerebral Aneurysms. Neurosurgery. 2018;82(5):638-644. doi:10.1093/neuros/nyx267

20. Edjlali M, Guédon A, Ben Hassen W, et al. Circumferential Thick Enhancement at Vessel Wall MRI Has High Specificity for Intracranial Aneurysm Instability. Radiology. 2018;289(1):181-187. doi:10.1148/radiol.2018172879

\section{SUPPLEMENTAL DIGITAL CONTENT}

Supplemental Digital Content. Table. Accuracy of the estimator of the specificity of AWE in our population, based on the hypothesis of 1000 included patients (binomial Clopper-Pearson approximation for $95 \%$ CI estimation). 
Supplemental Digital Content. Table. Accuracy of the estimator of the specificity of AWE in our population, based on the hypothesis of 1000 included patients (binomial Clopper-Pearson approximation for $95 \% \mathrm{Cl}$ estimation)

\begin{tabular}{|l|c|c|c|c|}
\hline $\begin{array}{l}\text { Number of not AWE and not } \\
\text { growth UIA }\end{array}$ & 600 & 700 & 800 & 900 \\
\hline Observed proportion (\%) & 60 & 70 & 80 & 90 \\
\hline $\begin{array}{l}95 \% \mathrm{Cl} \\
\text { of the proportion }\end{array}$ & {$[56.9-63.1]$} & {$[67.1-72.8]$} & {$[77.4-82.4]$} & {$[88-91.8]$} \\
\hline
\end{tabular}

